\title{
Alien plant species with a wider global distribution are better able to capitalize on increased resource availability
}

\author{
WayneDawson ${ }^{1,2}$,RudolfP.Rohr $\quad{ }^{3,4}$,MarkvanKleunen ${ }^{1,2}$ andMarkusFischer ${ }^{1}$

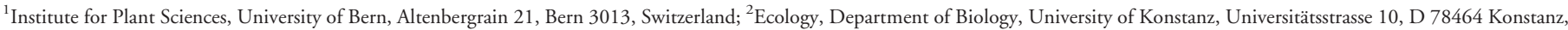 \\ Germany; ${ }^{3}$ Ecology and Evolution, Department of Biology, University of Fribourg, Chemin du Musée 10, Fribourg 1700, Switzerland; ${ }^{4}$ Integrative Ecology Group, Estación Biológica de \\ Doñana, CSIC, C/Américo Vespucio s/n, E-41092 Sevilla, Spain
}

Author of correspondence: WayneDawson Tel:+497531882116

Email:wayne.dawson@uni-konstanz.de

Keywords: exotic, fitness, life history, naturalization, phenotypic plasticity.

\section{Summary}

- A high ability of alien plant species to capitalize on increases in resource availability has been suggested as an explanation for being globally successful. Here, we tested this hypothesis meta-analytically using existing data from experiments manipulating plant resources (light, water and nutrients).

- From these studies we extracted the response to resource increase of biomass, as an indicator of plant performance, and the responses of two traits related to resource capture: root: shoot ratio and specific leaf area (SLA). For 211 species recorded in the GlobalCompendium ofWeeds, we assessed the relationship between effect sizes from such studies and the number of global regions where a species was established.

- We found that globally widespread species exhibited greater biomass responses to increases in resources overall, compared to less widespread species. Root: shoot ratio and SLA responses to increased resource availability were not related to species global distribution.

- In general, globally widespread alien plant species were better able to capitalize on increased availability of resources, through achieving increased growth and biomass accumulation, while greater plasticity of key resource-capture traits perse did not appear to be related to greater success.

\section{Introduction}

The ability of plants to increase performance by taking advantage of free resources may depend on plasticity of functional traits, in particular specific leaf area (SLA) and root : shoot ratio (Nicotra etal., 2010), and it has been suggested that such plasticity contributes to the invasion success of alien plants (Richards etal., 2006). Globally widespread alien species may exhibit high plasticity in physiological and morphological traits that contribute to the maintenance of plant performance under varying amounts of resources (Richards etal., 2006). Alternatively, successful species may exhibit plastic responses to increased resource availability that result in a higher plant performance in high-resource environments, whereas less successful alien or native co-occurring species are less plastic, and are thus less able to increase performance in high-resource environments (Richards etal., 2006). This second scenario might be a mechanism underlying the theory of fluctuating resource availability and invasibility (Davis etal., 2000), which suggests that the apparently idiosyncratic nature of invasion events results from a requirement of increased resource availability coinciding with invading propagule input.

In addition to biological invasions, increases in resource availability through habitat disturbance and eutrophication (e.g. atmospheric nitrogen deposition) are major elements of global environmental change (Sala etal. , 2000). It is possible that such increases in resource availability have been to the advantage of those alien, as well as native, species that can capitalize on extra resources. Such potential synergisms between elements of global change may have significant future implications for the extent of floral homogenization worldwide (Dukes \& Mooney, 1999; Bradley etal., 2010), if widespread alien species are able to further exploit such resource increases.

Several studies have tested experimentally how invasive plant species respond to changes in resource availability relative to native species (Funk, 2008; Funk \& Zachary, 2010) or noninvasive alien species (Burns, 2004; Hastwell \& Panetta, 2005; Schlaepfer etal., 2010). Such studies are at a local scale and consider several species, and in order to build wider generalizations the results of such studies can be combined in a quantitative meta-analysis (Davidson etal. , 2011; Palacio-Lopez \& Gianoli, 2011). However, many studies have been conducted that are not necessarily focused on invasions, and assess the responses of various plant species to increased availability of resources such as light (e.g. Reich etal., 1998), nutrients (e.g. James, 2008) and water (e.g. Sack \& Grubb, 2002). Such studies offer a valuable opportunity to assess whether more widespread alien plant species tend to be better than less widespread species in general at 
maintaining plant performance when resources are low, or at increasing performance when resource availability is high, and whether they differ in plastic responses of functional traits, such as SLA and root : shoot ratio. Moreover, use of data from studies that did not focus on invasions per se should minimize the potential for bias toward including only well-known or intensively studied invasive species that have been observed to become dominant after changes in resource availability in the field.

In this study, we employed a meta-analytical approach to test, across many studies and three resource types (nutrients, water and light), whether widespread alien plant species respond more strongly to increased resource availability than do less widespread species. Biomass offers the most direct measure of plant performance as a product of growth, and thus change in biomass should also offer the clearest indicator of a plant's ability to respond to and take advantage of increased resource availability. In this study, we combined published data on responses of biomass, root : shoot ratio and SLA of plant species to increased resource availability and a global measure of alien species distribution (Randall, 2002). Specifically, we asked whether:

- widespread species exhibit a greater increase in biomass in response to higher resource availability (nutrients, water and light) than do less widespread species;

- widespread species change root : shoot ratios to a greater extent than less widespread species in response to increased availability of below-ground resources (water and nutrients). Based on resource-allocation theory (Bloom etal. , 1985), we might expect that widespread species are better able to reduce root : shoot ratios in response to an increase in availability of below-ground resources, in order to adjust biomass allocation toward capture of light;

- widespread species decrease SLA to a greater degree than less widespread species in response to an increase in light availability. A greater reduction in SLA in high-light conditions would maintain a high photosynthetic capacity, while minimizing water loss, and thus allow for higher biomass production (Lusk etal. 2008).

Whilst two other meta-analyses have recently compared trait responses to increased resources of invasive, noninvasive and native species (Davidson etal., 2011; Palacio-Lopez \& Gianoli, 2011), our study is novel for three important reasons. First, we do not restrict our included studies and effect sizes to studies which focused on invasive species, allowing a larger number of effect sizes and species to be considered. Secondly, Palacio-Lopez \& Gianoli (2011) combined effect sizes of different traits together in the same analysis. However, there is no reason to expect success of species to be related to responses of all traits in the same way. Thirdly, we also incorporate phylogenetic structure in our analyses, as species more closely related to one another may respond more similarly to changes in availability of resources than species that are more distantly related to one another. Failure to consider the potential for phylogenetic nonindependence in such analyses could result in concluding that a significant relationship exists between responses to resource availability and global success when it is actually confounded by phylogenetic history.

\section{MaterialsandMethods}

\section{Study and data collection}

We executed a systematic search for data from existing studies published from 1990 to 2009 to obtain as many experimental studies as possible which had manipulated availabilities of light, nutrient or water received by plants, or some combination of the three resource types. Our search for studies was conducted using ISI Web of Science, and we entered as search terms all combinations of 'light'/'shade'/'nutrient*'/'nitrogen'/'drought'/'water' and 'plant phenotypic plasticity'/'plastic*'/'trait*'/'biomass'/ 'SLA'/'specific leaf area'/root : shoot ratio'. Once lists of potential studies had been compiled, we checked the text and figures of each study for evidence that the study involved a manipulative experiment (e.g. glasshouse study, application of resource treatment to planted plants), and for data on biomass (including above-ground only), root : shoot ratio and SLA responses to resource manipulation. We only included studies that reduced the likelihood of confounding resource availability treatments with genetic differences (i.e. studies using replicates of clones, half sibs or seed families in each resource treatment, and/or studies explicitly stating a randomized designation of treatments).

A total of 182 studies met these criteria, covering 347 species and yielding 547 treatment effect sizes. We extracted means, standard deviations and sample sizes of plant biomass, SLA and root : shoot ratio measures under a low-resource treatment and a high-resource treatment where available. We extracted the data from tables, from the main text or from graphs using the program ImageTool (University of Texas Health Centre, San Antonio, TX, USA). When studies had imposed more than two availabilities of a resource treatment, we took biomass in the lowest resource availability and the highest resource availability as 'low' and 'high' treatments, respectively. When studies had manipulated more than one resource type in an experiment, we always used the trait measures corresponding to manipulation of the target resource, when other resource types were highest and thus unlikely to be limiting. When experimental treatments had been applied to more than one population of a species, we extracted individual trait measures per population. We also recorded the type of resource manipulated (light, nutrients or water). Light manipulations involved shading, lighting, or manipulation of real canopies. Nutrient manipulations involved N, NP, NPK or organic material/soil addition. Water manipulations involved reduced provision of water as the 'low' resource treatment. We recorded the amount of the resource applied in the low- and high-resource treatments when available. For nutrients, we obtained the nitrogen concentration under nutrient manipulation treatments when NP or NPK or soil/organic matter were applied. We then calculated the magnitude of resource availability difference as the high-treatment amount divided by the low-treatment amount. Where available, we also extracted the duration of the experiment conducted (in d).

From the mean and standard deviations of trait measures and their sample sizes, we calculated each effect size as a log-response 
ratio and an associated variance following Rosenberg etal. (2000), with the low resource availability trait measure acting as 'control', and the high resource availability acting as 'treatment'. Thus, each species (or population within a species) within a study with a specific resource manipulation had an effect size representing the ratio of change in biomass, root : shoot ratio or SLA from a low to a high resource availability. A positive effect size would indicate an increase in the value of a trait, whilst a negative effect size would represent a decrease in the value of a trait.

\section{Global distribution of alien species}

Because the species from our study search have native ranges spread across the globe, we needed to use a global measure of distribution that integrates records of species present and established throughout their introduced ranges. We achieved this by using the GlobalCompendiumofWeeds (GCW; Randall, 2002), which has also been successfully used in other recent studies (Pyšek etal., 2009; Dawson etal. , 2011; Jenkins \& Keller, 2011). The GCW represents the most extensive compilation of invasive plant and weed species, incorporating 284 list references with global coverage, and cites $>18000$ taxa between them as being weedy, naturalized or invasive. Every species listed in the GCW is cited in at least one list reference as being in at least one of the above categories. Whilst the GCW is not $100 \%$ exhaustive, it provides the best indication to date of how globally widespread alien plant species are known to be, and allows comparison of species native to and introduced to different regions.

We derived a global measure of distribution from the GCW by first counting the number of list references within which a species was cited as 'alien', 'introduced', 'cultivation escape' or 'garden escape', 'naturalized', 'noxious weed', 'sleeper weed' or 'environmental weed'. Species listed in only one reference as a 'weed' were not included, as they may have only been recognized as weeds in the native ranges. From these references, we counted the number of global regions within which species were recorded. There were 11 regions in total (also see Dawson etal. , 2011): North America, South America, Central America (including the Caribbean), Europe, Africa, Middle East, South Asia (Bangladesh, Pakistan and India, and Sri Lanka), Eastern Asia (China, Taiwan, Japan, Nepal and Mongolia), Southeast Asia, Australasia and the Pacific Islands (including Hawaii i). It is difficult to discern whether or not species are truly invasive in all regions listed by the GCW. However, for species in our dataset, the number of global regions is well correlated with the number of states and provinces in North America where a species is naturalized (Spearman's $\rho=0.533, P<0.001$, number of species $=134$; North American data obtained from the United States Department of Agriculture (USDA) plant database, http://plants.usda.gov/java/). This North American distribution database has often been used as a proxy for invasiveness (Mitchell \& Power, 2003; van Kleunen \& Johnson, 2007). Thus, we believe the GCW represents a reasonable estimate of the extent to which alien species are established globally (i.e. they are not just introduced and under cultivation).

Out of the 347 species and 574 effect sizes covered by the resource-manipulation studies, there were 198 species (309 effect sizes) occurring in the GCW, which had sufficient data on resource manipulation and study duration. Species not occurring in the GCW may not have been widely introduced and cultivated outside of native ranges, or they may be noninvasive despite introduction. To distinguish these two groups of species, we checked for species records in the Germplasm Resources Information Network (GRIN) taxonomy online database (USDA ARS National Genetic Resources Program, 2010), and if the species was recorded there as cultivated, widely cultivated or naturalized, we included it in the final dataset. This yielded 13 species that were widely cultivated or recorded as naturalized but were not listed in the GCW, giving a total of 211 species (and 332 individual effect sizes) in the final dataset overall, across 129 studies (see Supporting Information, Methods S1). The extra 13 species were recorded as being in zero GCW regions. Most studies and species involved manipulation of nutrient availability (Table 1). Biomass responses were obtained for 149 species, whilst root : shoot ratio responses were obtained for 77 species, and SLA responses for 104 species (Table 1). The majority of species across all data sets were shrubs and trees, and forbs (Table 1). Most species were native to Eurasia and Africa (Table 1). Species with biomass and root : shoot ratio response effect sizes were represented mostly by forb and shrub/tree growth forms, and less by graminoids; species with SLA responses were mostly shrub and tree species (Table 1).

\section{Analysis}

In the analysis of the data we have to take into account two important features. First, we are dealing with a meta-analysis (Gurevitch \& Hedges, 1999). Secondly, our data points are related to species, and thus we have to take account of potential phylogenetic correlation, that is, the nonindependence of the data points induced by their common history (Felsenstein, 1985). The meta-analyses were conducted using the usual recommendations (Gurevitch \& Hedges, 1999) to take into account the between-effect size variance and the within-effect size variance. We weighted the data using the inverse of the within-effect size variance plus the estimation of between-effect size variance. For details about the computations, see chapters 16 and 20 of Borenstein etal. (2009).

The correlation induced by the phylogeny was incorporated into the analyses using phylogenetic regression (Grafen, 1989) with Pagel's lambda correlation structure (Freckleton etal., 2002). This involves using a parameter called lambda to adjust the strength of the correlation induced by Brownian motion, that is, the correlation between two species is given by lambda multiplied by the fraction of common time length on the phylogenetic tree. The higher lambda is, the greater the strength of the phylogenetic signal in the residuals; a lambda equal to zero implies that there is no phylogenetic correlation and a lambda equal to one is equivalent to a Brownian motion model. The construction of the phylogenetic trees was done using the online program Phylomatic (Webb \& Donoghue, 2005), and additional published phylogenies were used to resolve polytomies as much as possible (see Methods S2 for details). 
Table1 Summary of the number of studies and species for which effect sizes of plant-trait responses to increased resource availability could be extracted

\begin{tabular}{|c|c|c|c|c|c|c|c|c|}
\hline & \multicolumn{4}{|c|}{ Biomass } & \multicolumn{3}{|l|}{$\mathrm{R}: \mathrm{S}$} & \multirow{2}{*}{$\frac{\text { SLA }}{\text { Light }}$} \\
\hline & Light & Nutrient & Water & Overall & Water & Nutrient & Overall & \\
\hline Studies & 40 & 20 & 39 & 90 & 31 & 9 & 39 & 56 \\
\hline Effect sizes & 74 & 62 & 92 & 228 & 85 & 37 & 122 & 113 \\
\hline Species & 64 & 54 & 60 & 149 & 45 & 34 & 77 & 104 \\
\hline \multicolumn{9}{|l|}{ Growth form (species) } \\
\hline Graminoid & 8 & 14 & 11 & 28 & 9 & 2 & 10 & 6 \\
\hline Forb & 25 & 31 & 17 & 66 & 7 & 28 & 34 & 30 \\
\hline Liana & 2 & & 2 & 3 & 1 & & 1 & 4 \\
\hline Shrub/tree & 29 & 9 & 30 & 52 & 29 & 4 & 32 & 64 \\
\hline \multicolumn{9}{|l|}{ Origin (species) } \\
\hline Africa & & 2 & 3 & 5 & 3 & & 3 & 2 \\
\hline Asia & 8 & 3 & 9 & 17 & 9 & 1 & 10 & 19 \\
\hline Australasia & 1 & 1 & 1 & 3 & 1 & 1 & 2 & 3 \\
\hline Europe & 3 & 2 & 1 & 6 & 1 & 3 & 4 & 5 \\
\hline North America & 3 & 3 & 4 & 10 & 3 & 2 & 5 & 13 \\
\hline South America & 7 & 3 & 2 & 9 & 2 & & 2 & 7 \\
\hline Eurasia & 12 & 14 & 6 & 27 & 5 & 10 & 15 & 12 \\
\hline Africa + Eurasia & 19 & 23 & 22 & 51 & 15 & 15 & 29 & 24 \\
\hline North + South America & 2 & 1 & 2 & 4 & 1 & & 1 & 7 \\
\hline Disjunct/cosmopolitan & 9 & 2 & 10 & 17 & 5 & 2 & 6 & 12 \\
\hline
\end{tabular}

$\mathrm{R}: \mathrm{S}$, root : shoot ratio; SLA, specific leaf area. Also shown are the numbers of species of different growth forms and with different geographic origins represented by effect sizes of biomass, $R: S$ and SLA responses overall, and for each manipulated resource type.

For analysis of biomass responses, we included effect sizes from studies manipulating nutrient, water and light availability. For analysis of root : shoot ratio responses, we only included effect sizes from studies manipulating nutrient and water availability, because root : shoot ratio is a trait commonly measured in relation to uptake of below-ground resources. For analysis of SLA responses, on the other hand, we only included effect sizes from studies manipulating light, because SLA is a trait related to light capture and photosynthetic capacity (Wright etal., 2004). Moreover, for both root : shoot ratio and SLA, there were very few studies available for responses to changes in other resources.

Because in a meta-analytical context, the effect size has to be included as the dependent variable, we included our global distribution measure as an explanatory variable. This approach has the additional benefit that it allowed us to include other covariates in the models that represented important differences between studies in their methodology, which may affect the magnitude of effect sizes observed. Moreover, it also facilitated inclusion of a phylogenetic structure in our models. To test whether the trait responses depended on the resource type manipulated (nutrients, water or light), we included it as a categorical variable for biomass and root: shoot ratio responses (SLA had only one resource type). Because it is likely that the response of a trait increases with the magnitude of increase in resource availability, we included the magnitude of resource increase (the natural $\log$ of the high resource availability divided by the low resource availability) as a covariate. We also included duration of the study (natural-log transformed number of d) as a covariate. These two covariates and our measure of global distribution were scaled to their respective means and standard deviations to allow direct comparisons of model parameter estimates. For biomass and root : shoot ratio responses, we ran two separate models: one including an interaction term between resource type and global distribution, and a second without interactions between these two variables. This allowed us to assess the relationship between global distribution and species responses to resource increases across resource types, and the relationship between global success and species responses depending on the resource type.

The fit of the models was achieved within a Bayesian framework under R (R Development Core Team, 2009), and the phylogenies were manipulated using the $\mathrm{R}$ package 'ape' (Paradis etal. , 2004). We chose noninformative priors and the sampling of the posterior distribution was done using Monte Carlo Markov chains (burn-in of 5000, sampling of 300 000) (Gilks etal., 1996). The estimated values of the parameters were computed as the mean value of their corresponding chains, and we used the empirical confidence interval (at 95\%) as an estimate of the confidence intervals.

\section{Results}

\section{Biomass responses to increased availability of resources}

Biomass responses were significantly positive for all three resource types on average (Fig. 1a,b). Biomass responses did not significantly differ, on average, between the three resource types (Fig. 1a,b). As the difference between control and treatment resource availability increased ('resource increase'), the magnitude of biomass responses also significantly increased (Fig. 1a,b). The parameter estimate for the resource increase effect did not significantly differ between resource types, but the effect of increasing water availability on biomass responses tended to be smaller than increasing nutrient or light availability (Fig. 1a,b). 

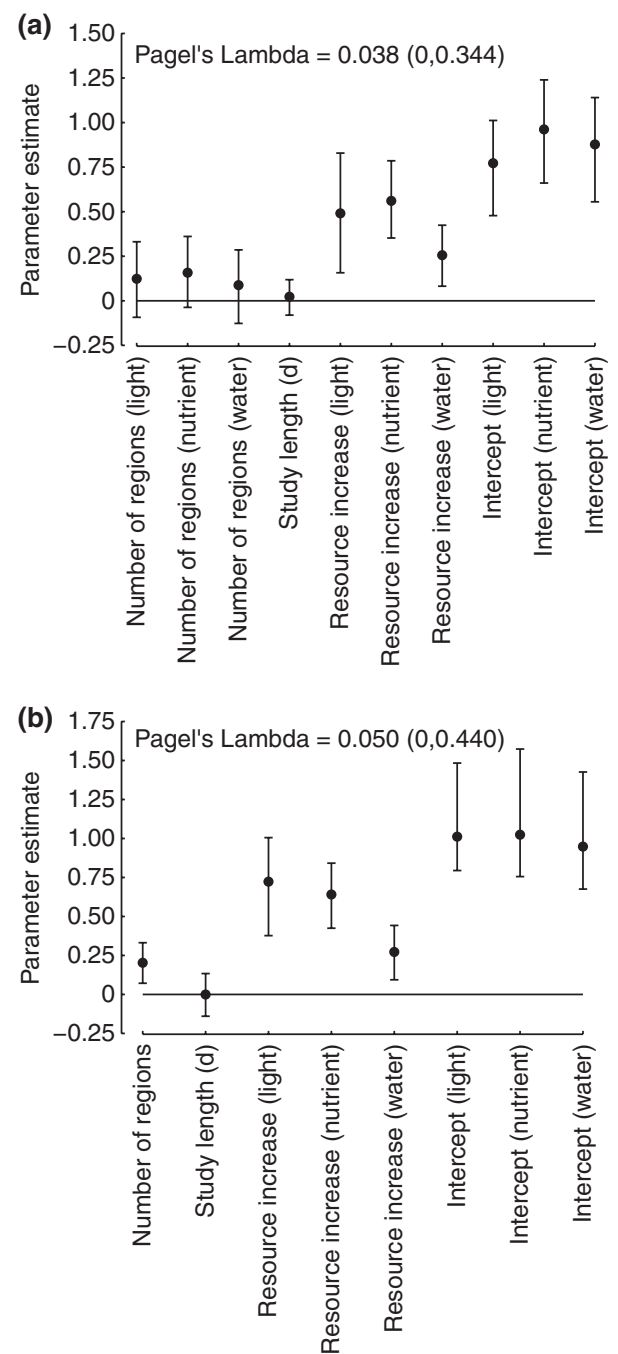

Fig. 1 The means (circles) and 95\% confidence intervals (bars) of parameter estimates describing the relationships between biomass response to increased resources and global distribution of alien species (number of regions), including: (a) interaction between number of regions and resource type; and (b) only main effects for number of regions and resource type. Positive estimates indicate an increase in biomass with increased resource availability for resource type (i.e. intercepts for light, nutrients and water), or a positive relationship between the magnitude of biomass increase and a continuous variable (amount of resource increase per resource type, study length (d), number of regions). Pagel's lambda with $95 \%$ confidence intervals is also given. The dotted line indicates an estimate of zero.

Study duration was not significantly related to the magnitude of biomass response (Fig. 1a,b). When our measure of species success (number of global regions) was allowed to interact with resource type in the model, biomass responses to increased resource availability were not significantly related to species success, for light, nutrients or water (Fig. 1a). However, across all three resource types (i.e. without an interaction between species success and resource type), biomass responses of species were significantly and positively associated with the number of global regions where a species was established (Fig. 1b). Pagel's lambda was low in both models, indicating there was little correlation structure induced by the phylogeny in the residuals of biomass
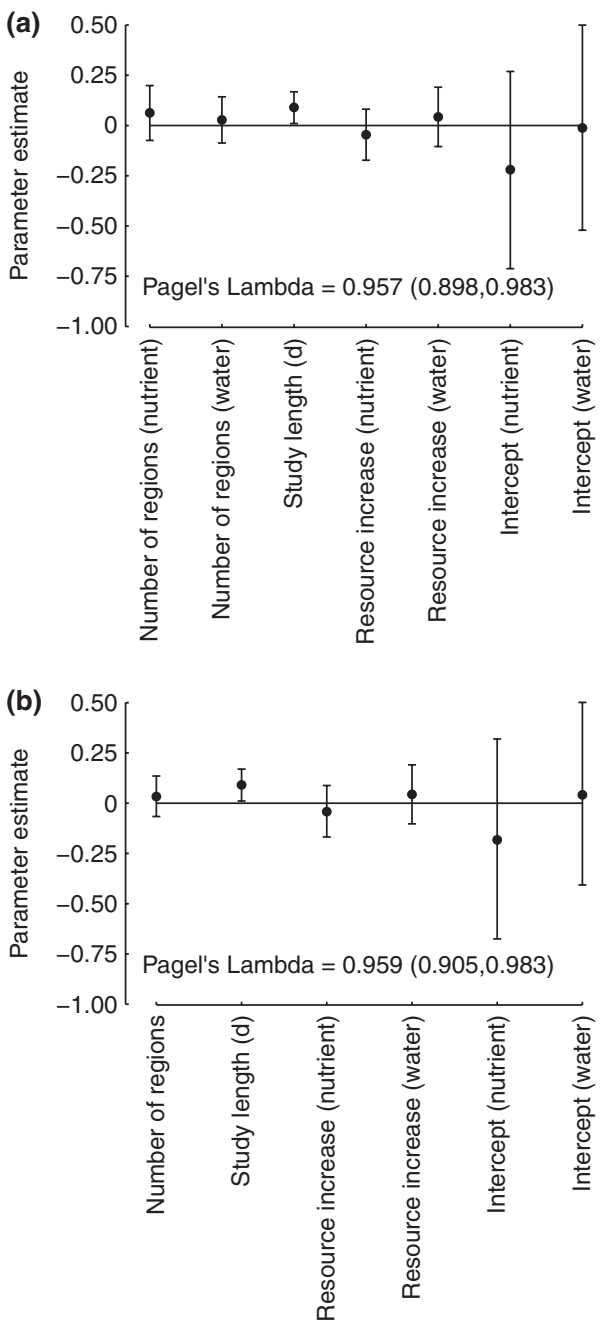

Fig. 2 The mean (circles) and 95\% confidence intervals (bars) of parameter estimates describing the relationships between root : shoot ratio response to increased resources and global distribution of alien species (number of regions), including: (a) interaction between number of regions and resource type; and (b) only main effects for number of regions and resource type. Positive estimates indicate an increase in root : shoot ratio with increased resource availability for resource type (i.e. intercepts for nutrients and water), or a positive relationship between the magnitude of root : shoot ratio increase and a continuous variable (amount of resource increase per resource type, study length (d), number of regions). Negative estimates indicate a decrease in root : shoot ratio. Pagel's lambda with $95 \%$ confidence intervals is also given. The dotted line indicates an estimate of zero.

responses, that is, there was almost no phylogenetic signal in biomass responses to increased resource availability and the number of global regions.

\section{Root : shoot ratio responses to increased availability of below-ground resources}

The intercepts for $\mathrm{R}$ : $\mathrm{S}$ responses to increased nutrient and water availability were not significantly different from zero, suggesting that, on average, $R: S$ ratios did not significantly change with increased nutrient or water availability (Fig. 2a,b). In addition, $\mathrm{R}: \mathrm{S}$ responses also did not significantly change with increasing 
differences in resource availability between control and treatment (Fig. 2a,b). The variation in root : shoot ratio responses to increased water and nutrient availabilities was large (see 95\% credible intervals in Fig. 2a,b), indicating that biomass allocation shifts under changing resource availabilities are likely to be species-specific.

Study duration was significantly and positively related to the degree of $\mathrm{R}: \mathrm{S}$ response. As the intercepts did not significantly differ from zero, this indicates that with increasing time, the reduction in $R$ : $S$ in response to increased resource availability becomes smaller (the R : $\mathrm{S}$ of control and treatment plants converge with increasing experiment duration). The response of $\mathrm{R}$ : $S$ to increased resource availability was not significantly related to the number of global regions occupied, for either nutrient increase or water increase (Fig. 2a). There was also no significant relationship between the number of global regions and $\mathrm{R}: \mathrm{S}$ responses overall (Fig. 2b). The value for lambda was close to one (Fig. 2a,b), indicating that changes in root : shoot ratios with increased resource availability were strongly associated with evolutionary history.

\section{SLA responses to increased availability of light}

The intercept of SLA response was significantly negative, indicating, as expected, that across studies and species, increased light intensities led to a decrease in SLA. As the magnitude of resource increase from control to treatment plants grew larger, the magnitude of SLA reduction also increased, as indicated by the significantly negative parameter estimate for resource increase (Fig. 3). Responses of SLA to increased light availability were not significantly related to the number of regions occupied by a species, or to the duration of the study (Fig. 3). Pagel's lambda was very

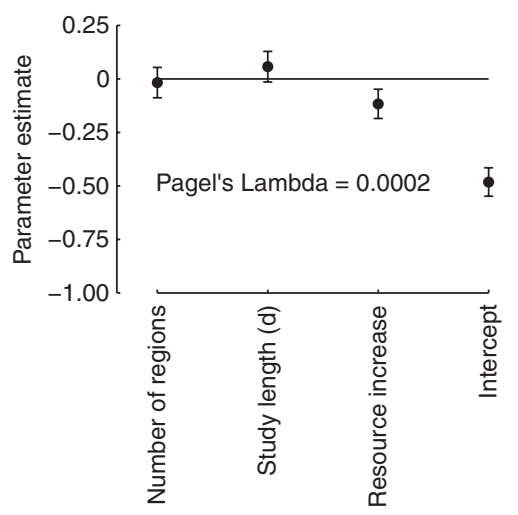

Fig. 3 The means (circles) and 95\% confidence intervals (bars) of parameter estimates describing the relationships between specific leaf area (SLA) response to increased light intensities and global distribution of alien species (number of regions), and the covariates; amount of resource increase and study length (d). The intercept represents the mean effect size (at average values for other scaled, continuous variables). The negative estimate for the intercept indicates a decrease in SLA with increased light availability. The negative estimate for amount of resource increase indicates a greater SLA decrease with increasing light availability. Pagel's lambda was very close to zero. The dotted line indicates an estimate of zero. close to zero, suggesting that there was almost no phylogenetic signal in SLA responses to increased light availability.

\section{Discussion}

Recently, increasing theoretical attention has been paid to the potential role of phenotypic plasticity in species traits in facilitating the spread of invasive alien species by maintaining plant performance under varying environmental conditions, or through allowing species to maximize performance when resource availability is increased (Richards etal., 2006). The results of our meta-analysis of large numbers of studies manipulating resources for single or few species suggest that species that are more globally widespread are better at capitalizing on increased resource availability, when light, nutrients and water are considered together. Thus, among alien plants, globally successful species are not those best able to maintain plant performance in varied environments, but tend to be those that are best able to utilize the increases in resource availability that typify human-disturbed habitats worldwide (Richards etal. , 2006; Pyšek \& Richardson, 2007). The lack of a significant relationship between biomass responses and global distribution when resources are considered separately may reflect lower statistical power, as the trends all tended to be positive. Our results support those of Davidson etal. (2011), who found that when studies compared invasive alien plant species with native species, the alien species tended to be more plastic in their biomass responses to increased resource availability than the native species. Gonzalez etal. (2010) also found that invasive plant species increased performance and growth rate to a greater extent than native species, under increased nutrient availability. For this response to be a true advantage, globally successful alien species should also be more responsive to increased resources than less successful alien species (van Kleunen etal. , 2010), and our findings indicate that this is likely to be the case.

Our results may also corroborate the theory of fluctuating resource availability and invasibility, if invasion depends upon alien species responding strongly to increased resource availability. Davis etal. (2000) suggested that species are only able to colonize and establish in a community if resource availability increases, either through resource input or by reduced resource uptake by the community. An extension of this theory is that species better able to use an increase in resource availability may be more successful at establishing than those that are less able to respond. Leishman \& Thomson (2005) found that increases in nutrient availability were crucial in allowing invasive alien plants to establish over low-fertility adapted natives in areas of disturbance with big increases in water availability, but with inherently low fertility. It is unclear from our study whether globally successful species tend to establish in open, disturbed habitats with low amounts of competition, or whether they are also able to establish in less disturbed, competitive communities when resource availabilities are increased. Many studies in our analyses did not test the responses of species to resource increase in a community context, which would be a prerequisite for testing both the theory of fluctuating resource availability and the hypothesis 
that greater alien responses increase the likelihood of the alien invading a community. We cannot rule out the possibility that globally distributed species are merely those best suited to establishing in open, anthropogenically altered habitats, which also tend to have elevated amounts of resources. Thus, the global distributions of successful species may simply reflect the worldwide distribution of similarly disturbed, resource-enriched environments.

In less disturbed habitats, the importance of an increase in resources for establishment of alien plants is likely to vary, depending on how historically limiting the resources in question are at a particular location (Davis \& Pelsor, 2001), and on how the recipient community responds to increased resource availability relative to incoming aliens. Increased availability of a limiting resource is only likely to reduce the amount of competition and community resistance if growth responses by the community do not result in other resources becoming limited. For example, without adequate disturbance, nutrient additions may increase the competition intensity for light exerted by the resident community (Hautier etal. , 2010) in areas where soils are relatively fertile, thus increasing, rather than decreasing, resistance to invasion. Moreover, it has recently been argued that factors affecting the ability of alien species to establish may act in concert rather than in isolation, such that patterns of invasion success in relation to single factors alone are not apparent (Blumenthal, 2005). Local-scale studies that set species' responses to increased resource availability in a community context, and interacting with other factors such as enemy release (Blumenthal etal., 2009), will better elucidate how different responses among species play out in terms of shifts in abundance and dominance of alien and native species in those communities. However, notwithstanding interactions with resident communities and other biotic and abiotic factors, our study still indicates that globally successful species tend to be more suited to take advantage of increases in resource availability than less successful species.

Optimal-resource allocation theory (Bloom etal., 1985) predicts that plants should invest biomass into structures that allow for uptake of the most limiting resource. However, we found that plants, on average, did not reduce biomass allocation to roots when below-ground resources increased. This contrasts with the results of Reynolds \& D'Antonio (1996) and Poorter et al. (2012), who showed that root mass ratios across multiple species decreased on average in response to increased nutrient availability. Moreover, there was no relationship between root: shoot ratio responses to increased resource availability and global distribution. This corroborates further the findings of Reynolds \& D'Antonio (1996), and more recently Poorter etal. (2012), in that species best suited to high-nutrient environments did not alter their root mass ratios in response to increased nutrient availability compared to species from low-nutrient environments. However, Davidson etal. (2011) found that invasive alien species tended to be more plastic in root: shoot ratio responses to increased resource availability than native species. Whilst aliens may be better than neighbouring natives at shifting biomass allocation from roots to shoots under more favourable resource availability (Davidson etal. , 2011), our results suggest that at a global scale an ability to change biomass allocation may not offer a consistent advantage to alien species.

In line with expectations of adaptive shade-tolerance plasticity (Valladares \& Niinemets, 2008), SLA decreased when light availability increased, and this decrease tended to be stronger when the difference in light availability increased between treatment and control plants. However, in contrast to expectations, widespread species did not decrease SLA to a greater extent than did less widespread species when light availability was higher. A recent multi-species study, including 14 invasive and noninvasive congeneric pairs of species, also found no difference between invasive and noninvasive species in SLA responses to light (van Kleunen etal. , 2011). Similarly, plasticity in SLA did not differ between invasive alien and native species in the meta-analysis by Davidson etal. (2011). This implies that plasticity in the ability of plants to capture more light under both low- and high-light conditions by changing SLA does not necessarily contribute to success at a global scale.

The presence of a relationship between global success and biomass responses, but the absence of a relationship with root : shoot ratio and SLA responses is intriguing. The greater biomass increases of more widespread alien species under higher availability of resources may be the result of changes in resource-capture and physiological traits other than SLA and root : shoot ratio. Alternatively, the performance of plants in varied environments may not simply be the product of their ability to change individual traits (Funk, 2008), but may involve varied responses by multiple traits (Richards etal. , 2006). Moreover, resource-acquisition traits of successfully invading species are often dependent on the type of habitat being invaded (Leishman etal. , 2010; Tecco etal. , 2010). Differences (Ordonez etal. , 2010; Godoy etal. , 2011) or similarities (Leishman etal., 2010; Tecco etal., 2010) between aliens and natives may be more important to alien success than differences in the capacity of traits to vary (Godoy etal. , 2011). Alternatively, Palacio-Lopez \& Gianoli (2011) suggest that establishment success of newly introduced species in wide-ranging environments may be determined more by rapid local adaptation than by plasticity. In any case, direct and consistent correlations between plasticity of single traits and plant performance or global success seem increasingly unlikely. Focusing on the endpoint of trait plasticity - the performance or fitness of the plant under different environmental conditions - is perhaps a more useful approach to understanding whether or not globally widespread or invasive species are successful owing to their ability to survive and outperform other species in multiple environments.

In summary, our study suggests that the ability of plant species to increase biomass in response to increased resource availability also appears to confer species success at a global scale, but that plasticities of individual resource-capture traits are unlikely to be consistently related to species success. The search for a relationship between plasticity of individual traits and species success may be less fruitful than measuring plant performance under different environments. Whether an ability to increase biomass translates ultimately to increased individual and population-level fitness for widespread, successful species requires more detailed experiments involving multiple native and alien, successful and 
unsuccessful species, grown under different environmental conditions and involving direct fitness measurements.

\section{Acknowledgements}

This work was funded by the Swiss National Science Foundation in the framework of the Swiss National Centre for Competence in Research 'Plant Survival'. R.P.R. was also partially supported by funding from FP7-REGPOT 2010-1, grant no. 264125 EcoGenes.

\section{References}

BloomAJ,ChapinFS,MooneyHA.1985 . Resource limitation in plants - an economic analogy. AnnualReviewofEcologyerSystematics 16:363-392.

BlumenthalD.2005 . Inter-related causes of plant invasion. Science 310: 243-244.

BlumenthalD,MitchellCE,Pys `ekP,JarosikV.2009 . Synergy between pathogen release and resource availability in plant invasion. Proceedingsofthe NationalAcademyofSciences, USA 106: 7899-7904.

BorensteinM,HedgesLV,HigginsJPT,RothsteinHR.2009 . Introductionto meta-analysis. Chichester, UK: John Wiley \& Sons, Ltd.

BradleyBA,BlumenthalDM,WilcoveDS,ZiskaLH.2010 . Predicting plant invasions in an era of global change. TrendsinEcologyandEvolution 25: $310-318$

BurnsJH.2004 . A comparison of invasive and non-invasive dayflowers (Commelinaceae) across experimental nutrient and water gradients. Diversity andDistributions 10: 387-397.

DavidsonAM,JennionsM,NicotraAB.2011 . Do invasive species show higher phenotypic plasticity than native species, and if so, is it adaptive? A meta-analysis. EcologyLetters 14: 419-431.

DavisM,PelsorM.2001 . Experimental support for a resource-based mechanistic model of invasibility. EcologyLetters 4: 421-428.

DavisMA,GrimeJP,ThompsonK.2000 . Fluctuating resources in plant communities: a general theory of invasibility. JournalofEcology 88: $528-534$.

DawsonW,FischerM,vanKleunenM.2011 . Maximum relative growth rate of common UK plant species is positively associated with their global invasiveness. GlobalEcologyandBiogeography 20: 299-306.

DukesJS,MooneyHA.1999 . Does global change increase the success of biological invaders? TrendsinEcologyandEvolution 14: 135-139.

FelsensteinJ.1985 . Phylogenies and the comparative method. American Naturalist 125: 1-15.

FreckletonRP,HarveyPH,PagelM.2002 . Phylogenetic analysis and comparative data: a test and review of evidence. AmericanNaturalist 160 : $712-726$

FunkJL.2008 . Differences in plasticity between invasive and native plants from a low resource environment. JournalofEcology 96: 1162-1173.

FunkJL,ZacharyVA.2010 . Physiological responses to short-term water and light stress in native and invasive plant species in southern California. Biological Invasions 12: 1685-1694.

GilksWR,RichardsonS,SpiegelhalterDJ,eds.1996 . Markovchainmontecarlo inpractice. Boca Raton, FL, USA: Chapman \& Hall.

GodoyO,ValladaresF,Castro-DiezP.2011 . Multispecies comparison reveals that invasive and native plants differ in their traits but not in their plasticity. FunctionalEcology 25: 1248-1259.

GonzalezAL,KominoskiJS,DangerM,IshidaS,IwaiN,RubachA.2010 . Can ecological stoichiometry help explain patterns of biological invasions? Oikos 119: 779-790.

GrafenA.1989 . The phylogenetic regression. PhilosophicalTransactionsofthe RoyalSociety,LondonSeriesB-BiologicalSciences 326: 119-157.

GurevitchJ,HedgesLV.1999 . Statistical issues in ecological meta-analyses. Ecology 80: 1142-1149.

HastwellGT,PanettaFD.2005 . Can differential responses to nutrients explain the success of environmental weeds? JournalofVegetationScience 16:77-84.
HautierYL,NiklausPA,HectorA.2010 . Competition for light causes plant biodiversity loss after eutrophication. Science, 324: 636-638.

JamesJJ.2008 . Effect of soil nitrogen stress on the relative growth rate of annual and perennial grasses in the Intermountain West. PlantandSoil 310: 201-210.

JenkinsC,KellerSR.2011 . A phylogenetic comparative study of preadaptation for invasiveness in the genus Silene (Caryophyllaceae). BiologicalInvasions 13: $1471-1486$.

vanKleunenM,DawsonW,SchlaepferDR,JeschkeJM,FischerM.2010 . Are invaders different? A conceptual framework of comparative approaches for assessing determinants of invasiveness. EcologyLetters, 13: 947-958.

vanKleunenM,JohnsonSD.2007 . Effects of self-compatibility on the distribution range of invasive European plants in North America. Conservation Biology 21: 1537-1544.

vanKleunenM,SchlaepferDR,GlaettliM,FischerM.2011 . Pre-adapted for invasiveness: do species traits or their plastic responses to shading differ between invasive and non-invasive plant species in their native range? Journalof Biogeography 38: 1294-1304.

LeishmanMR,ThomsonVP.2005 . Experimental evidence for the effects of additional water, nutrients and physical disturbance on invasive plants in low fertility Hawkesbury Sandstone soils, Sydney, Australia. JournalofEcology 93: $38-49$.

LeishmanMR,ThomsonVP,CookeJ.2010 . Native and exotic invasive plants have fundamentally similar carbon capture strategies. JournalofEcology 98 : $28-42$.

LuskCH,ReichPB,MontgomeryRA,AckerleyDD,Cavender-BaresJ.2008 Why are evergreen trees so contrary about shade? TrendsinEcologyand Evolution 23: 299-303.

MitchellCE,PowerAG.2003 . Release of invasive plants from fungal and viral pathogens. Nature, 421: 625-627.

NicotraAB,AtkinOK,BonserSP,DavidsonAF,FinneganEJ,MathesiusU, PootP,PuruggananMD,RichardsCL,ValladaresF etal. 2010. Plant phenotypic plasticity in a changing climate. TrendsinPlantScience 15 : 684-692.

OrdonezA,WrightIJ,OlffH.2010 . Functional differences between native and alien species: a global-scale comparison. FunctionalEcology 24: 1353-1361.

Palacio-LopezK,GianoliE.2011 . Invaisve plants do not display greater phenotypic plasticity than their native or non-invasive counterparts: a meta-analysis. Oikos 120: 1393-1401.

ParadisE,ClaudeJ,StrimmerK.2004 APE: analyses of phylogenetics and evolution in R language. Bioinformatics 20: 289-290.

PoorterH,NiklasKJ,ReichPB,OleksynJ,PootP,MommerL.2012 . Biomass allocation to leaves, stems and roots: meta-analyses of interspecific variation and environmental control. NewPhytologist 193: 30-50.

PyšekP,Jaros `íkV,PerglJ,RandallR,Chytry ' M,Kü hnI,Tichy' L,DanihelkaJ, ChrtekjunJ,Sa 'dloJ.2009. The global invasion success of central European plants is related to distribution characteristics in their native range and species traits. DiversityandDistributions 15: 891-903.

PyšekP,RichardsonDM.2007 . Traits associated with invasiveness in alien plants: where do we stand? In: Nentwig W, ed. Biologicalinvasions. Berlin, Heidelberg, Germany: Springer-Verlag, 97-126.

RDevelopmentCoreTeam . 2009. R:alanguageandenvironmentforstatistical computing. Vienna, Austria: R Foundation for Statistical Computing.

RandallRP.2002 . Aglobalcompendiumofweeds . Melbourne, Vic., Australia: RG and FJ Richardson.

ReichPB,TjoelkerMG,WaltersMB,VanderkleinDW,BushenaC.1998 Close association of RGR, leaf and root morphology, seed mass and shade tolerance in seedlings of nine boreal tree species grown in high and low light. FunctionalEcology 12: 327-338.

ReynoldsHL,D'AntonioCD.1996 . The ecological significance of plasticity in root weight ratio in response to nitrogen: opinion. PlantandSoil 185: 75-97.

RichardsCL,BossdorfO,MuthNZ,GurevitchJ,PigliucciM.2006 . Jack of all trades, master of some? On the role of phenotypic plasticity in plant invasions. EcologyLetters 9: 981-993.

RosenbergM,AdamsDC,GurevitchJ.2000 . MetaWinversion2:statistical softwareformeta-analysiswithresamplingtests . Sunderland, MA, USA: Sinauer Associates. 
SackL,GrubbPJ.2002 . The combined impacts of deep shade and drought on the growth and biomass allocation of shade-tolerant woody seedlings. Oecologia 131: $175-185$.

SalaOE,ChapinFS,ArmestoJJ,BerlowE,BloomfieldJ,DirzoJ,

Huber-SanwaldE,HuennekeLF,JacksonRB,KinzigA etal. 2000. Global biodiversity scenarios for the year 2100. Science 287: 1770-1774.

SchlaepferDR,GlaettliM,FischerM,vanKleunenM.2010 . A multi-species experiment in their native range indicates pre-adaptation of invasive alien plant species. NewPhytologist 185: 1087-1099.

TeccoPA,DiazS,CabidoM,UrcelayC.2010 . Functional traits of alien plants across contrasting climatic and land-use regimes: do aliens join the locals or try harder than them? JournalofEcology 98: 17-27.

UniversityofTexasHealthScienceCenter . 2002. UTHSCAImageToolversion 3.0. San Antonio, TX, USA: University of Texas Health Science Center. [WWW document]. URL. http://ddsdx.uthscsa.edu/dig/download.html. [accessed on 10 January 2010].

USDAARSNationalGeneticResourcesProgram2010 . GermplasmResources InformationNetwork-(GRIN) [Online Database]. Beltsville, MD, USA: National Germplasm Resources Laboratory. [WWW document]. URL http://www.ars-grin.gov/cgi-bin/npgs/html/taxgenform.pl?language=en [accessed on 15 June 2010].

ValladaresF,NiinemetsU ${ }^{*}$.2008 . Shade tolerance, a key plant feature of complex nature and consequences. AnnualReviewofEcologyandSystematics 39 : $237-257$.
WebbCO,DonoghueMJ.2005 . Phylomatic: tree assembly for applied phylogenetics. MolecularEcologyNotes 5: 181-183.

WrightIJ,ReichPB,WestobyM,AckerlyDD,BaruchZ,BongersF,

Cavender-BaresJ,ChapinT,CornelissenJHC,DiemerM etal. 2004. The worldwide leaf economics spectrum. Nature 428: 821-827.

\section{SupportingInformation}

Additional supporting information may be found in the online version of this article.

MethodsS1 List of studies from which data were extracted.

Methods S2 Methods for constructing phylogenetic trees, and trees used.

Please note: Wiley-Blackwell are not responsible for the content or functionality of any supporting information supplied by the authors. Any queries (other than missing material) should be directed to the NewPhytologist Central Office. 